\title{
Excellent 10-year patient-reported outcomes and survival in a single- radius, cruciate-retaining total knee arthroplasty
}

\author{
Chloe E. H. Scott ${ }^{1}\left[\right.$ ] $\cdot$ Katrina R. Bell ${ }^{1} \cdot{\text { Richard T. } \mathrm{Ng}^{2} \cdot \text { Deborah J. MacDonald }}^{1} \cdot$ James T. Patton $^{1} \cdot$ Richard Burnett $^{1}$
}

Received: 13 June 2018 / Accepted: 20 September 2018 / Published online: 1 October 2018

(c) The Author(s) 2018

\begin{abstract}
Purpose Over 2 million Triathlon single-radius total knee arthroplasties (TKAs) have been implanted worldwide. This study reports the 10-year survival and patient-reported outcome of the Triathlon TKA in a single independent centre.

Methods From 2006 to 2007, 462 consecutive cruciate-retaining Triathlon TKAs were implanted in 426 patients (median age 69 (21-89), 289 (62.5\%) female). Patellae were not routinely resurfaced. Patient-reported outcome measures (SF-12, Oxford Knee Scores (OKS), satisfaction) were assessed preoperatively and at 1, 5 and 10 years when radiographs were reviewed. Forgotten Joint Scores (FJS) were collected at 10 years. Kaplan-Meier survival analysis was performed.

Results At 10-11.6 years, 123 patients (128 TKAs) had died and 8 TKAs were lost to follow-up. There were four aseptic failures (two cases of tibial loosening, two cases of instability) and four septic failures requiring revision. Symptomatic aseptic radiographic loosening was present in three further cases at 11 years. Four (1\%) patellae were secondarily resurfaced. OKS score improved by $17.7 \pm 9.7$ points at 1 year $(p<0.001)$, and was maintained at $34.7 \pm 9.6$ at 10 years with FJS $48.5 \pm 31.4$. Patient satisfaction was $88 \%$ at each timepoint. Ten-year survival was $97.9 \%$ (95\% confidence interval 96.5-99.3) for revision for any reason, $98.9 \%(97.7-100)$ for mechanical failure, and $98.6 \%$ (97.4-99.8) for aseptic loosening (symptomatic radiographic or revised).

Conclusion The Triathlon TKA continues to show excellent longer-term results with high implant survivorship, low rates of aseptic failure, consistently maintained PROMs and excellent patient satisfaction rates of $88 \%$ at 10 years.

Level of evidence II, Prospective cohort study.
\end{abstract}

Keywords Total knee arthroplasty $\cdot$ Single-radius knee $\cdot$ Long-term survival $\cdot$ Patient-reported outcomes

\section{Introduction}

The number of total knee arthroplasties (TKAs) performed annually in the United Kingdom continues to rise with projections suggesting this is to continue at least to 2035 in the United Kingdom [12] and internationally [27, 31]. Though TKA is a cost-effective treatment for end-stage degenerative joint disease [29], patient dissatisfaction rates of 15-20\% are consistently reported [3,36]. Dissatisfaction is highest in younger patients $(<55$ years) [35] who at present are both

Chloe E. H. Scott

chloe.scott@nhslothian.scot.nhs.uk

1 Department of Orthopaedics, Royal Infirmary of Edinburgh, 51 Little France Crescent, Old Dalkeith Road, Edinburgh EH16 4SA, UK

2 Department of Orthopaedics, University of Calgary, 401 9th Ave SW, Suite 335, Calgary, AB T2P3C5, Canada the fastest growing utilisers of TKA and have the highest revision rates $[31,37]$. There are therefore both patientcentred and population-centred drivers to improve functional outcome in TKA. Though predictors of outcomes are complex and multifactorial, implant design is potentially significant.

The design of condylar resurfacing TKA femoral components has followed the theory of a dynamic flexion-extension axis (FEA) following a J-shaped curve throughout a range of motion [18] since 1976 [28]. More recent cadaveric $[6,25]$ and three-dimensional imaging studies $[16,26]$ have suggested an alternative flexion-extension axis at the knee, common throughout a range of motion. This common FEA approximates to the surgical epicondylar axis $[1,16]$ and has a consistent relationship with both the patellofemoral joint axis [11] and the longitudinal rotational axis of the tibia [26]. It is a consistent feature in both varus and valgus knees [26]. This modern kinematic theory has been adopted 
in single-radius implant designs since 1996 and has more recently been combined with deep flexion adaptations in the Triathlon TKA. Though over 2 million Triathlon TKAs have now been implanted worldwide, no independent reports of mid- to long-term survival or outcomes of this design are yet published.

A single common flexion-extension axis at the knee conveys several theoretical biomechanical advantages. Ligament isometry throughout the range of movement facilitates more conforming polyethylene thus reducing contact stresses in addition to reducing mid-flexion instability. The more posterior location of the common FEA compared to J-shaped axes, lengthens the quadriceps moment arm providing a theoretical mechanical advantage to knee extension power [20] with a reduction in joint reaction force at the patellofemoral joint. Additional features of the Triathlon TKA include shorter posterior condyles with consistent posterior condylar offset between sizes to encourage deep flexion.

In 2016 the Triathlon TKA was the most common TKA prosthesis implanted in Australia and the third most implanted in the United Kingdom. Despite this, to date there have been no independent studies of mid- to long-term functional outcomes, radiographic reviews or details of complications other than revision which is proved in registry data. Ten-year survival is 96.1 (95\% confidence interval (95\% CI) 95.5-96.6) for the cruciate retaining Triathlon in the Australian registry 96.8\% (95\% CI 96.3-97.2) in the National Joint Registry of England and Wales. Good survival and functional outcomes have previously been reported at 5 years [34]. The aim of this study was to report the 10-year survival of the Triathlon TKA from a single independent centre. Secondary aims included radiographic assessment and patientreported outcome measures (PROMs) of function and pain to determine the longitudinal outcomes over 10 years.

\section{Materials and methods}

From 2006 to 2007, data were recorded for consecutive patients undergoing Triathlon TKAs (Stryker Orthopaedics, Mahwah, NJ, USA) performed or supervised by seven consultant surgeons at a single large orthopaedic teaching hospital. Cemented, cruciate-retaining TKAs with standard tibial baseplates were performed in all cases via a medial parapatella approach using a tourniquet. Simplex bone cement (Stryker Orthopaedics, Mahwah, NJ, USA) was used both with $(n=349)$ and without $(n=113)$ gentamicin. The patella was not routinely resurfaced. The patella was resurfaced primarily in 24 patients (5.2\%) at the surgeons' discretion. Antibiotic prophylaxis was with three doses cefuroxime. Standard primary implants were used in all but one patient where a medial tibial plateau fracture non-union required medial augmentation and tibial stem. A CR implant was used in this case. Four TKAs were navigated (surgeon discretion). Of the 36 patients who underwent bilateral TKAs, 9 patients had 18 TKAs performed as bilateral simultaneous procedures. All patients followed standardised postoperative rehabilitation with mobilisation from day 1 and discharge home when independently mobile with two sticks.

\section{PROMs}

Prior to surgery, a postal questionnaire including the Shortform (SF-12) general health questionnaire [15] and the knee-specific Oxford Knee Score [13] (OKS) was sent to all patients. This was collected in a pre-assessment clinic 3 weeks prior to surgery. The SF-12 is a validated generic health questionnaire with physical and mental health components. Following TKA the minimal clinically important difference (MCID) in the physical component score is 4.5 for pain and 4.8 for function [8]. The OKS is a validated knee-specific outcome measure of 12 questions with 5 possible answers giving a score from 0 to 48 [13] and a MCID of 5 [8]. Higher scores represent better function. Completed questionnaires were collected at a nurse-led pre-assessment clinic.

Post-operative questionnaires were sent to patients at 0.5 , 1, 5 and 10 years. Response rates are detailed in Fig. 1. In addition to SF-12 and Oxford Knee Scores, questionnaires at and beyond 1 year included measures of patient satisfaction. Patients were asked, 'How satisfied are you with your operated knee?' with options 'very satisfied', 'satisfied', 'unsure' or 'dissatisfied' [36]. At 5 and 10 years patients were asked if they had undergone any reoperations and the nature of these. At 10 years the Forgotten Joint Score (FJS) was also collected. The FJS is a validated PROM outcome measure following knee arthroplasty designed to minimise ceiling effects in high-functioning individuals following knee arthroplasty [21]. Collection of data was independent of the routine clinical care of the patient. Patients who did not respond to the 5- and 10-year questionnaires were contacted by telephone and completed the questionnaire verbally.

\section{Survival}

Medical and operation notes were examined for all patients. The patients' demographics, indication, consultant in charge of care, date of surgery, and side were recorded. All intraoperative, early or late complications and their nature were recorded. Deep infection was defined as clinical infection with an identified organism; cellulitis was defined as superficial erythema with no apparent joint involvement which resolved with oral antibiotics; wound dehiscence was secondary gaping of part of the surgical wound requiring surgical intervention; and prolonged wound leakage was leakage which resolved in the absence of signs suggesting deep 


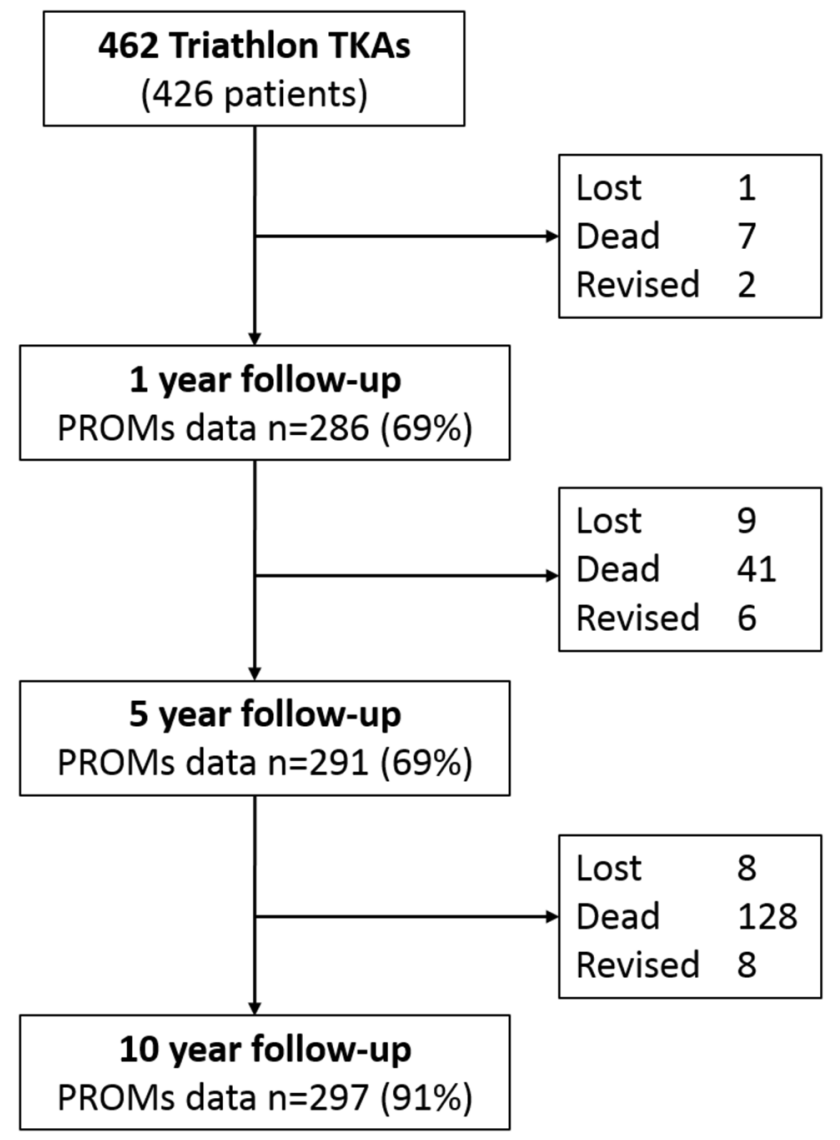

Fig. 1 Study cohort with follow-up at each timepoint. All figures in the boxes on the right are cumulative

infection (pain, decreased range of motion, sepsis, organism on aspiration). In those patients who had undergone revision surgery, the mode of implant failure confirmed at revision was noted. Any other reoperation was also noted. Deceased patients were identified and date of death confirmed.

\section{Radiographic review}

Short-leg weight-bearing radiographs taken at most recent orthopaedic review were examined by two independent reviewers (KRB and RTN) who had no clinical contact with the patients. Coronal and sagittal implant alignment was measured to one decimal place and has been reported previously [34]. Periprosthetic radiolucencies were reported in femoral and tibial regions as per the Knee Society Score [17]. If radiolucencies were present, all radiographs pertaining to that TKA were independently examined by both reviewers to assess progression. Where $>2$ radiolucencies or any ballooning osteolysis was present, radiographs were additionally reviewed by a senior author (CEHS). The coronal and sagittal plane implant alignment have been reported previously [34].
Ethical approval was obtained for this prospective cohort study (Scotland (A) Research Ethics Committee 16/SS/0026). This study was completed without external funding.

\section{Statistical analysis}

Data were analysed using SPSS version 21.0. Interobserver agreement of radiolucencies was tested using the Kappa statistic. Repeated measures ANOVA was used to examine changes in parametric variables over the 10-year study period. A $p$ value of $<0.05$ was considered to be statistically significant. Post hoc analysis of longitudinal PROMs was performed using paired $t$ tests for parametric variables and Wilcoxon signed rank for non-parametric variables with significance set at $p<0.01$ incorporating a Bonferroni correction to adjust for multiple testing at 5 points over 10 years. Survival analysis was undertaken with life-tables and Kaplan-Meier analysis. The endpoints used were revision for any reason, mechanical failure (aseptic loosening and instability), aseptic failure (including symptomatic radiographic and revision for aseptic loosening), and a worst-case-scenario analysis assuming that all TKAs lost to follow-up had failed.

\section{Results}

In the study period, 462 consecutive cruciate retaining Triathlon TKAs were implanted in 426 patients. Median age was 69 years (range 21-89) and 289 (62.5\%) were female. The indication for surgery was primary osteoarthritis in 406/462 (87.9\%).

During the study (Fig. 1), 123 patients (128 TKAs (27.7\%)) had died with their implant intact. At minimum follow-up of 10 years (mean 10.8, SD 0.38) PROMs were obtained for 297 of 326 remaining TKAs (91\%). Eight patients (8 TKAs $(1.7 \%)$ ) were uncontactable and were considered lost. One patient uncontactable at 5 years was located at 10 years. Other non-responders $(n=21)$ included $11(2.4 \%)$ patients with dementia and $10(2.2 \%)$ who were contacted, but refused to complete questionnaires though had intact TKAs.

\section{Complications and survival analysis}

Early ( $<6$ weeks) and late complications ( $>6$ weeks) and reoperations are detailed in Table 1. Deep infections were managed with debridement and implant retention in three cases (two with long-term antibiotic suppression) and four with revision. There were four other revisions during the study period undertaken for mechanical reasons, including two cases of tibial aseptic loosening. Radiographic 
Table 1 Complications

\begin{tabular}{lc}
\hline Complication & Number $(\%)$ \\
\hline Early & \\
Prolonged wound leak & $19(4.1)$ \\
Wound dehiscence & $1(0.2)$ \\
Cellulitis & $2(0.4)$ \\
Deep infection & $1(0.2)$ \\
VTE & $9(1.9)$ \\
Myocardial infarction & $3(0.6)$ \\
Late & \\
Infection & $7(1.5)$ \\
Instability & $6(1.3)$ \\
Tibial loosening & $2(0.4)$ \\
Periprosthetic fracture & $3(0.6)$ \\
Anterior knee pain & $19(4.1)$ \\
Unexplained pain & $10(2.2)$ \\
Stiffness & $2(0.4)$ \\
Reoperations (not revision) & \\
All & $21(4.5)$ \\
Secondary wound closure & $1(0.2)$ \\
Debridement and implant retention & $3(0.6)$ \\
Manipulation under anaesthesia & $10(2.2)$ \\
Arthrolysis & $1(0.2)$ \\
Secondary resurfacing & $4(0.9)$ \\
Open reduction and internal fixation & $2(0.4)$ \\
\hline
\end{tabular}

VTE venous thromboembolism

review identified three additional cases of symptomatic radiographic tibial loosening. The life table for all revisions is given in Table 2. Individual failures are detailed in Table 3. Ten-year Kaplan-Meier survival analyses are shown in Table 4 and Fig. 2.

\section{Radiographic outcome}

Mean medial proximal tibial angle was $89.8^{\circ} \pm 1.9^{\circ}$; posterior tibial slope $2.7^{\circ} \pm 2.3^{\circ}$; femoral valgus angle $2.0^{\circ} \pm 1.9^{\circ}$; and femoral component flexion $15.7^{\circ} \pm 4.1^{\circ}$ as previously published [34]. Radiographic review at mean $8.22 \pm 1.94$ years of 266 TKAs identified: 1 case of definite symptomatic radiographic failure (tibial loosening with osteolysis); 9 concerning for loosening (2 symptomatic-3 tibial only, 5 femoral only, 1 both); 11 with $>2$ zones [17] of lucency and 105 with $\leq 2$ zones [17] of lucency consistent with cementation defects (zones 1 and 4 tibia or femur).

\section{Patient-reported outcome measures}

SF-12 physical component scores $(p<0.001)$ changed significantly over the study period (Table 5 ) with the greatest change in the first 6 months: $30.2 \pm 7.15$ to $41.5 \pm 10.0$ $(p<0.001)$. Mental component scores did not change significantly over the study period $(p=0.014)$.

Oxford Knee Scores changed significantly over the study period ( $p<0.001$, Table 5; Fig. 3). The greatest improvement in mean population scores was in the first 6 months: $18.8 \pm 6.9$ to $34.3 \pm 10.1(p<0.001)$. A statistically significant decline occurred between 5 and 10 years $[37.3 \pm 10.3$ to $34.7 \pm 10.5(p<0.001)]$ though this was less than the MCID. Mean improvement in OKS for individuals was $17.2 \pm 9.7$ at 1 year $(p<0.001)$ and $15.7 \pm 11.5$ at 10 years. The mean Forgotten Joint Score (FJS) was $48.2 \pm 33.7$ at 10 years (Fig. 4).

Patient satisfaction was $88.3 \%$ at 1 year, $88.0 \%$ at 5 years and $88.4 \%$ at 10 years. Of 35 patients dissatisfied at 5 years, 7 became satisfied by 10 years. Similarly, 9 who were satisfied at 5 years, were dissatisfied at 10 . OKSs were worse in those dissatisfied at 10 years [20.3 \pm 8.6

Table 2 Life table for total knee arthroplasty failures requiring revision

\begin{tabular}{|c|c|c|c|c|c|c|c|c|c|}
\hline \multirow[t]{2}{*}{ Interval (years) } & \multirow[t]{2}{*}{ Number } & \multirow[t]{2}{*}{ Failures } & \multirow[t]{2}{*}{ Lost } & \multirow[t]{2}{*}{ Withdrawn } & \multirow[t]{2}{*}{ At risk } & \multirow{2}{*}{$\begin{array}{l}\text { Failure } \\
\text { rate }(\%)\end{array}$} & \multirow{2}{*}{$\begin{array}{l}\text { Cumulative } \\
\text { survival (\%) }\end{array}$} & \multicolumn{2}{|l|}{$95 \% \mathrm{CI}$} \\
\hline & & & & & & & & Lower limit & Upper limit \\
\hline $0-1$ & 462 & 2 & 1 & 7 & 458 & 0.4 & 99.6 & 99.0 & 100 \\
\hline $1-2$ & 452 & 1 & 0 & 4 & 450 & 0.2 & 99.4 & 99.0 & 99.8 \\
\hline $2-3$ & 447 & 0 & 1 & 5 & 444 & 0 & 99.4 & 99.0 & 99.8 \\
\hline $3-4$ & 441 & 0 & 0 & 5 & 438 & 0 & 99.4 & 99.0 & 99.8 \\
\hline $4-5$ & 436 & 1 & 2 & 15 & 427 & 0.2 & 99.2 & 98.8 & 99.6 \\
\hline $5-6$ & 418 & 2 & 0 & 12 & 412 & 0.5 & 98.7 & 98.1 & 99.3 \\
\hline $6-7$ & 404 & 0 & 2 & 14 & 396 & 0 & 98.7 & 98.1 & 99.3 \\
\hline $7-8$ & 388 & 0 & 1 & 12 & 381 & 0 & 98.7 & 98.1 & 99.3 \\
\hline $8-9$ & 375 & 1 & 1 & 21 & 364 & 0.3 & 98.4 & 97.8 & 99.0 \\
\hline $9-10$ & 352 & 1 & 0 & 23 & 340 & 0.3 & 98.1 & 97.5 & 98.7 \\
\hline $10-11$ & 328 & 0 & 0 & 213 & 221 & 0 & 98.1 & 97.5 & 98.7 \\
\hline$>11$ & 115 & 0 & 0 & 115 & 57 & 0 & 98.1 & 97.5 & 98.7 \\
\hline
\end{tabular}

CI confidence interval 
Table 3 Details of individual total knee arthroplasty failures
Table 4 Ten-year KaplanMeier survival functions for different end points

\begin{tabular}{llllll}
\hline Age & Sex & Indication & Survival (years) & Mode of failure & Management \\
\hline 74 & M & OA & 0.25 & Infection & Two-stage revision \\
82 & M & OA & 0.33 & Infection & DAIR and long-term suppression \\
57 & M & OA & 0.5 & Infection & Two-stage revision \\
62 & M & OA & 1.67 & Infection & Two-stage revision \\
71 & F & \#NU & 3.75 & Infection & DAIR and long-term suppression \\
61 & F & OA & 4.3 & Aseptic loosening tibia & Revision \\
55 & F & RA & 5.25 & Aseptic loosening tibia & Revision \\
52 & F & RA & 5.8 & Instability & Revision \\
74 & F & OA & 8.6 & Infection & Two-stage revision \\
45 & F & OA & 9.6 & Instability & Revised \\
\hline
\end{tabular}

$M$ male, $F$ female, $O A$ osteoarthritis, $R A$ rheumatoid arthritis, \# fracture, $N U$ non-union, DAIR debridement and implant retention

\begin{tabular}{lcl}
\hline End point & $n$ & Survival \% (95\% CI) \\
\hline Any revision & 8 & 97.9 (96.5-99.3) \\
Mechanical failure (aseptic loosening or instability) & 4 & 98.9 (97.7-100) \\
Aseptic loosening (revised or symptomatic radiographic) & 5 & $98.6(97.4-99.8)$ \\
Any reoperation (including revisions) & 29 & $93.0(90.5-95.6)$ \\
Worst-case scenario (revised or lost) & 23 & $94.4(92.1-96.8)$ \\
\hline
\end{tabular}

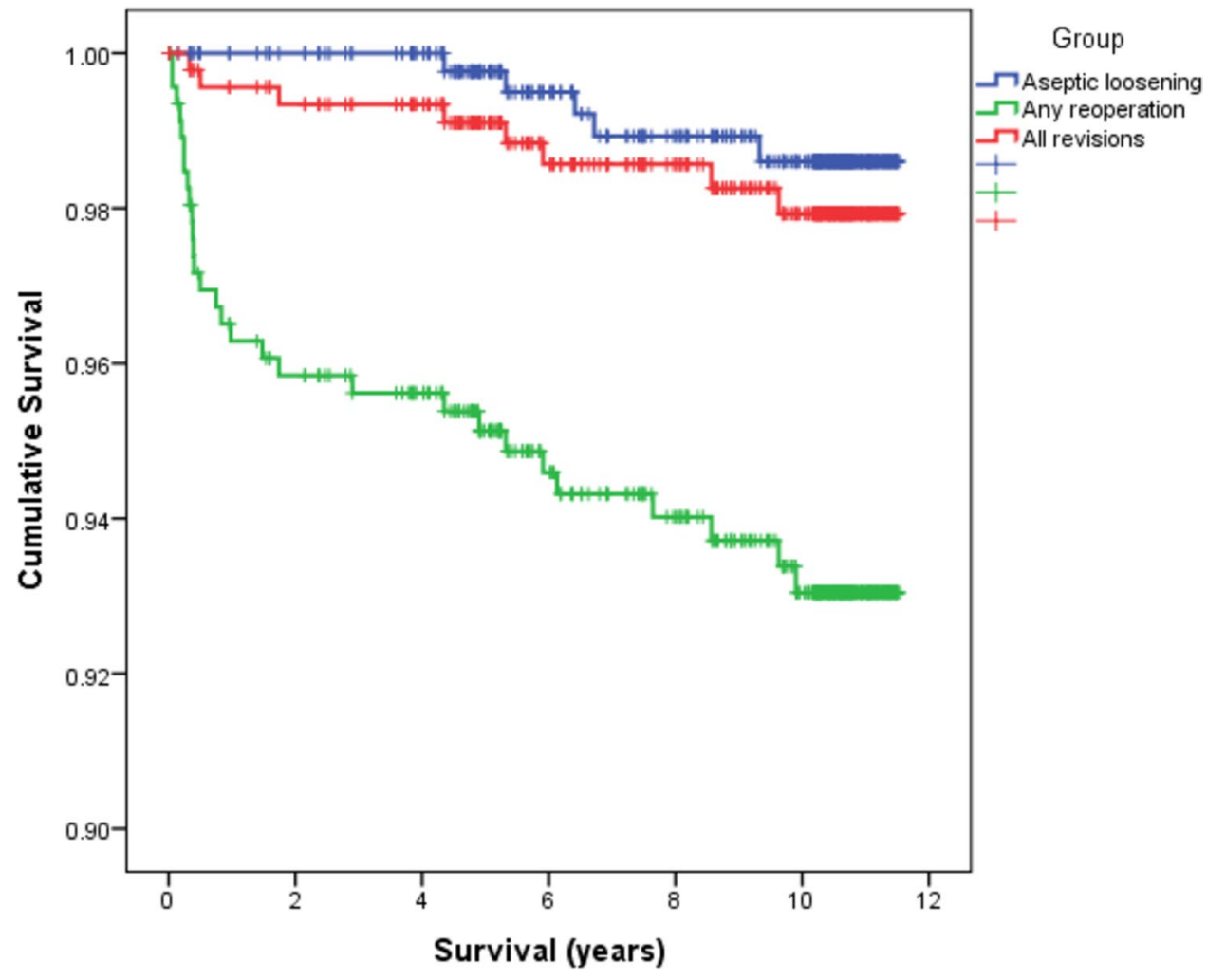


Table 5 Absolute PROMs at each timepoint with improvements in OKS for individuals

\begin{tabular}{lllll}
\hline PROM & Timepoint & Median & Mean $(95 \%$ CI $)$ & $p$ value \\
\hline PCS & Preop & 29.0 & $30.5(29.3$ to 31.8$)$ & $<0.001^{\wedge}$ \\
& 0.5 year & 42.1 & $41.5(39.8$ to 43.1$)$ & \\
1 year & 43.4 & $43.4(41.5$ to 45.2$)$ & \\
5 years & 39.3 & $41.7(39.7$ to 43.8$)$ & \\
& 10 years & 39.6 & $39.2(37.2$ to 41.2$)$ & \\
MCS & Preop & 53.6 & $51.3(49.4$ to 53.2$)$ & \\
& 0.5 year & 55.4 & $52.3(50.6$ to 54.0$)$ & \\
& 1 year & 55.9 & $52.6(50.9$ to 54.4$)$ & \\
& 5 years & 54.4 & $51.7(49.9$ to 53.5$)$ & \\
OKS & 10 years & 50.9 & $48.6(46.7$ to 50.5$)$ & \\
& Preop & 18 & $18.8(17.6$ to 19.9$)$ & \\
& 0.5 year & 37 & $34.3(32.6$ to 36.1$)$ & \\
& 1 year & 39 & $36.3(34.6$ to 38.0$)$ & \\
& 5 years & 41 & $37.3(35.5$ to 39.0$)$ & $<0.001^{*}$ \\
OKS improvement & 10 years & 38 & $34.7(32.9$ to 36.5$)$ & $<0.001^{*}$ \\
& Preop to 1 year & 18 & $17.7(16.1$ to 19.2$)$ & \\
& 1-5 years & 1 & $1.2(-0.1$ to 2.4$)$ & \\
& 5-10 years & -1.5 & $-3.0(-4.3$ to -1.7$)$ & \\
\end{tabular}

$p$ values ( ${ }^{\wedge}$ two-way ANOVA, *paired $t$ tests) reflect changes over time between values

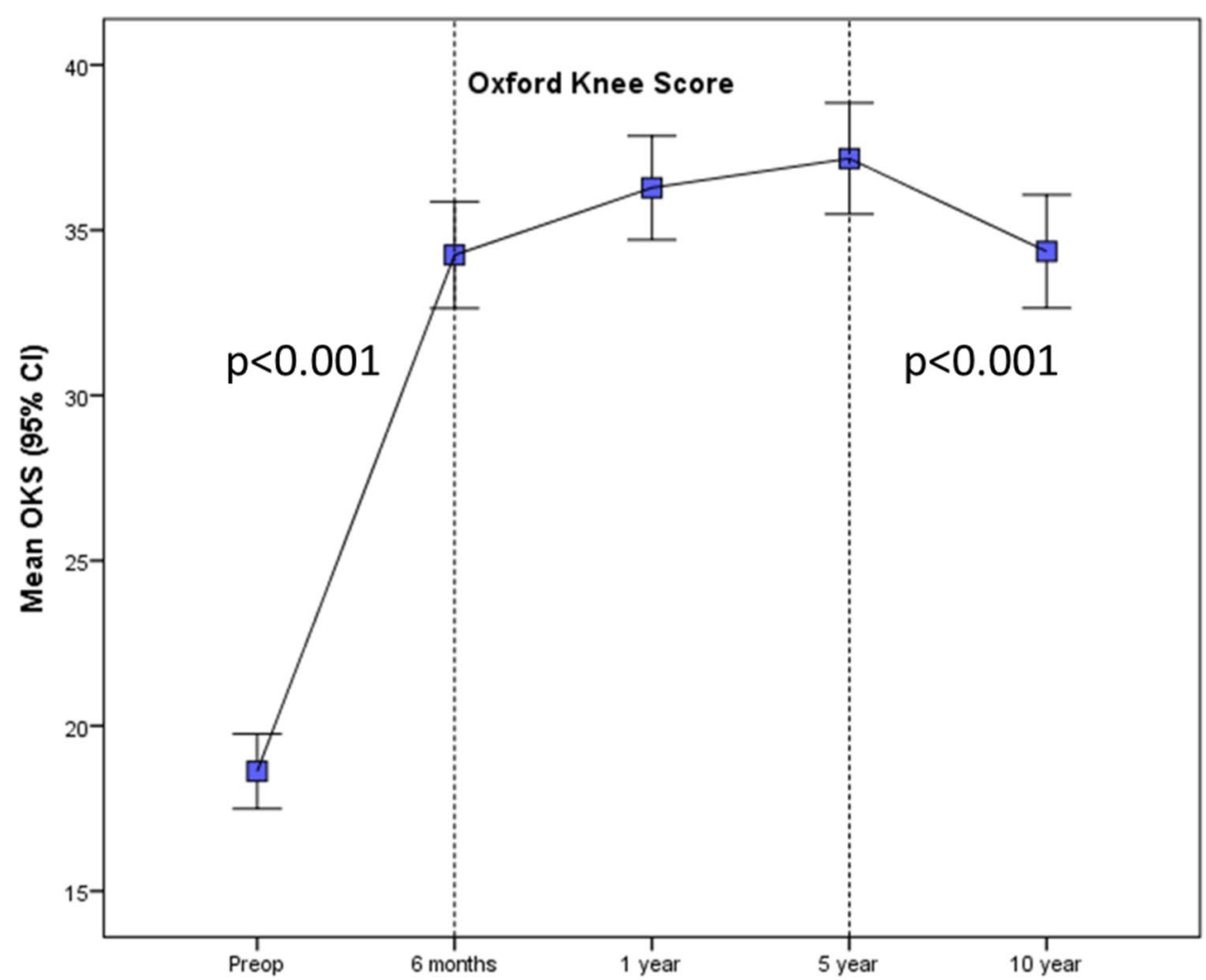

Fig. 3 Longitudinal Oxford Knee Scores (OKS) over the 10 -year study period. Significant improvement occurred over the first year with a small but significant decline from 5 to 10 years 
Fig. 4 The Forgotten Joint Score (FJS) at 10 years

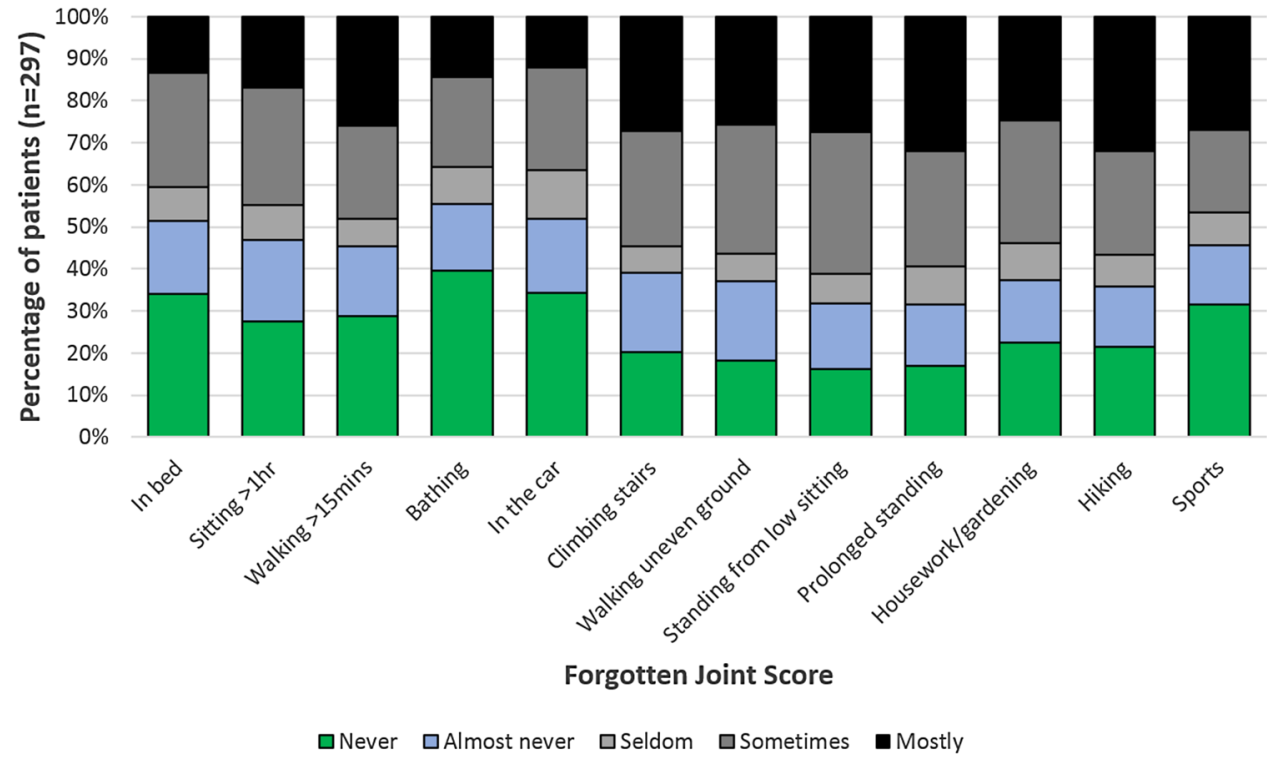

versus $35.2 \pm 9.8(p<0.001)]$. Nineteen patients $(19$ TKAs, $4 \%$ ) reported anterior knee pain at clinical followup, one persistent after secondary resurfacing.

\section{Discussion}

The key findings of this study are the high patient satisfaction $(88 \%)$ and well maintained improvements in functional outcome scores at 10 years following a single-radius cruciate-retaining TKA with all cause survival of 97.9\% (95\% CI 96.5-99.3) and a worst-case analysis of 94.4\% (92.1-96.8). This level of satisfaction compares well with the existing literature where satisfaction rates of $80-85 \%$ are consistently reported $[3,36]$. The survival analysis is consistent with that reported by the National Joint Registry of England and Wales where a 10-year survival of $96.8 \%$ (96.3-97.2) is reported for 61,067 cemented cruciate retaining Triathlon TKAs, equivalent to eighth among 28 brands of TKA. Though joint registries report absolute implant survival with large sample sizes, this is not the only metric of implant success, or failure, and cohort studies offer beneficial complementary analysis including data on reoperations, modes of failure, radiographic loosening and PROMs. To date this is the only independent 10-year study of this popular implant of which $>2$ million have been implanted worldwide to date. Very few patients were lost to follow-up in this study and long-term PROMs were obtained for $91 \%$ of those patients alive with intact TKAs at 10 years.

The mean individual improvement in OKS found in this study of $17.2 \pm 9.7$ by 1 year is favourable compared to the National PROMs database for England and Wales where a mean improvement in OKS 16.52 points at 6 months in 40,841 patients with $56 \%$ data capture is reported following primary TKA. Studies of longitudinal PROMs following TKA are scarce. Williams et al. [39] reported OKSs in 1547 TKA patients over 10 years demonstrating a mean preoperative OKS of 19.5 (95\% CI 18.8-20.2) in a population with similar demographics improving to $34.3(33.5-35.1)$ at 1 year, peaking at 2 years and then slowly declining to 30.1 (29.1-31.1) at 10 years. They present population means at each time-point, rather than the improvements experienced by individual patients. Both population and individual OKS improvements here compare favourably with these scores. To limit any potential ceiling effects in high-functioning individuals, and to investigate the performance of this TKA in returning to specific functions, the Forgotten Joint Score was added to the 10-year follow-up questionnaire. This identified that patients were most aware of their arthroplasty during hiking, walking on uneven ground, when standing for a prolonged time, when standing from a low sitting position and when climbing stairs.

Satisfaction remained high throughout follow-up with $88 \%$ satisfied or very satisfied at every timepoint. This is favourable when compared to satisfaction rates of $81 \%$ consistently reported in the literature $[3,5,36]$. Changes in satisfaction over time have rarely been examined. Nilsdotter et al. [32] studied 102 patients over 5 years finding satisfaction to be unchanged from 1 to 5 years. Though overall rates remained the same in our study, 17/291 (5.8\%) patients changed satisfaction status from 1 to 5 years, and 16/297 (5.4\%) changed from 5 to 10 years. This phenomenon has also been reported by Clement et al. [7] who identified three groups of dissatisfied patients following TKA: those with early dissatisfaction at 1 year only; those with persistent dissatisfaction at 5 years; and those with late dissatisfaction only. All dissatisfied patients at 10 years in our study 
reported pain, usually diffusely. Pain has frequently been identified as an important predictor of dissatisfaction $[3,36]$.

The single-radius concept has performed well in joint registry data since it was introduced in the Scorpio TKA in 1996 with a 10-year risk of revision of 3.9\% (95\% CI 3.7-4.3). Though the current study lacks a multi-radius control group, single and multi-radius TKAs have been compared previously. Randomised control trials comparing the Triathlon single-radius TKA with multi-radius TKAs have found in favour of the single-radius design [9, 20, 22]. Hamilton et al. [22] reported significantly greater range of motion, more rapid recovery to $116 \%$ of quadriceps function compared to the contralateral side, greater satisfaction and reduced 'worst daily pain' following Triathlon $(n=104)$ compared to a multi-radius TKA $(n=108)$ over the 3 years of follow-up [22]. Collados-Maestre et al. [9] found significantly better knee society scores, WOMAC pain scores, range of motion and quadriceps strength in the single-radius group when comparing 118 single-radius and 119 multi-radius TKA designs at 5-7 years [9]. Biomechanical studies comparing single and multi-radius TKAs have found larger amounts of axial rotation in single-radius knees using dynamic fluoroscopy [19] and better power absorption during weight acceptance in single-radius TKAs at gait analysis [19]. These studies appear to support the patellofemoral friendly nature of single-radius designs. In our study, primary patella resurfacing was performed in 24 patients $(5.2 \%)$, a rate markedly less than the $52 \%$ at reported for Triathlon TKAs in the NJR [2]. Four additional patients underwent secondary resurfacing for persistent anterior knee pain, one of whom continued to report anterior knee pain at 10 years. Anterior knee pain was reported clinically by $4 \%$ of patients. This is favourable compared to rates of $19-40 \%$ reported in the literature for patients following TKA $[4,38]$. With no control group it is impossible to comment on whether the theoretically favourable patellofemoral kinematics of this implant with a longer quadriceps moment arm translate into clinical benefit. The most common reason for reoperation was knee stiffness which required MUA in ten cases. This incidence is consistent with previous reports of stiffness following TKA of 1-5\% [30].

Non-randomised studies comparing outcomes of the Triathlon TKA to other multi-radius designs including the Duracon [10,33] and the Kinemax Plus [14] have found in favour of the single-radius design in terms of pain relief [10, $14,33]$, stability [10], degree of flexion [10, 33], walking and stair climbing ability [10], Knee Society Scores [33] and knee-related quality of life [14]. Favourable Knee Society Scores have been reported by Harwin et al. [23] in 2035 Triathlon TKAs in 1688 patients with 48-point improvements for pain and 22 for function at a mean of 21 months. Other non-randomised studies have found no differences in PROMs between single- and multi-radius posterior-stabilised TKAs [24].
The limitations of this study include a lower preoperative OKS response rate than at other assessment timepoints. As with all studies utilising PROM questionnaires, not all patients answer all the questions which can lead to responder bias. Attempts have been made to restrict any bias by including and reporting all available data in the analysis. Range of motion data were not collected and radiographs were not available for all patients. Radiographs were not all taken at the same timepoint reflecting the reality of clinical practice, though the most recent was used for analysis. Limitations are mitigated by only $8 / 462(1.7 \%)$ TKAs being lost- to followup with validated PROMs on $93 \%$ of surviving patients at this timepoint and presentation of a worst-case-scenario analysis.

\section{Conclusion}

This is the first independent study of 10-year survivorship and patient-reported outcome following cruciate-retaining single-radius TKA. It provides the first non-registry evidence for a widely used implant. It confirms that the good joint registry survival is coupled with excellent radiographic and functional outcomes and high patient satisfaction. The single-radius design theory utilised in this implant translates to a durable TKA prosthesis with favourable functional outcome and excellent patient satisfaction of $88 \%$.

Acknowledgements This research did not receive any specific grant from funding agencies in the public, commercial, or not-for-profit sectors. The authors thank all of the orthopaedic surgeons whose patients were included in this study. The authors acknowledge the financial support of NHS Research Scotland (NRS), through Chloe Scott of NHS Lothian.

Funding This study was completed without external funding.

\section{Compliance with ethical standards}

Conflict of interest Author JTP and author RB have received financial support for education from Stryker. Authors CEHS, KRB, RTN and DM have no conflicts of interest to disclose.

Ethical approval All procedures performed in studies involving human participants were in accordance with the ethical standards of the institutional and/or national research committee (Scotland A Research Ethics Committee) and with the 1964 Helsinki declaration and its later amendments or comparable ethical standards.

Open Access This article is distributed under the terms of the Creative Commons Attribution 4.0 International License (http://creativeco mmons.org/licenses/by/4.0/), which permits unrestricted use, distribution, and reproduction in any medium, provided you give appropriate credit to the original author(s) and the source, provide a link to the Creative Commons license, and indicate if changes were made. 


\section{References}

1. Asano T, Akagi M, Nakamura T (2005) The functional flexionextension axis of the knee corresponds to the surgical epicondylar axis: in vivo analysis using a biplanar image-matching technique. J Arthroplast 20:1060-1067

2. Baker PN, Petheram T, Dowen D, Jameson SS, Avery PJ, Reed MR et al (2014) Early PROMs following total knee arthroplastyfunctional outcome dependent on patella resurfacing. J Arthroplast 29:314-319

3. Baker PN, van der Meulen JH, Lewsey J, Gregg PJ, National Joint Registry for England and Wales (2007) The role of pain and function in determining patient satisfaction after total knee replacement. Data from the National Joint Registry for England and Wales. J Bone Jt Surg Br 89:893-900

4. Baliga S, McNair CJ, Barnett KJ, MacLeod J, Humphry RW, Finlayson D (2012) Does circumpatellar electrocautery improve the outcome after total knee replacement? A prospective, randomised, blinded controlled trial. J Bone Jt Surg Br 94:1228-1233

5. Bourne RB, Chesworth BM, Davis AM, Mahomed NN, Charron KD (2010) Patient satisfaction after total knee arthroplasty: who is satisfied and who is not? Clin Orthop Relat Res 468:57-63

6. Churchill DL, Incavo SJ, Johnson CC, Beynnon BD (1998) The transepicondylar axis approximates the optimal flexion axis of the knee. Clin Orthop Relat Res 356:111-118

7. Clement ND, Bardgett M, Weir D, Holland J, Gerrand C, Deehan DJ (2018) Three groups of dissatisfied patients exist after total knee arthroplasty: early, persistent, and late. Bone Jt J 100-B:161-169

8. Clement ND, MacDonald D, Simpson AH (2014) The minimal clinically important difference in the Oxford knee score and Short Form 12 score after total knee arthroplasty. Knee Surg Sports Traumatol Arthrosc 22:1933-1939

9. Collados-Maestre I, Lizaur-Utrilla A, Gonzalez-Navarro B, Miralles-Munoz FA, Marco-Gomez L, Lopez-Prats FA et al (2017) Better functional outcome after single-radius TKA compared with multi-radius TKA. Knee Surg Sports Traumatol Arthrosc 25:3508-3514

10. Cook LE, Klika AK, Szubski CR, Rosneck J, Molloy R, Barsoum WK (2012) Functional outcomes used to compare single radius and multiradius of curvature designs in total knee arthroplasty. $\mathrm{J}$ Knee Surg 25:249-253

11. Coughlin KM, Incavo SJ, Churchill DL, Beynnon BD (2003) Tibial axis and patellar position relative to the femoral epicondylar axis during squatting. J Arthroplast 18:1048-1055

12. Culliford D, Maskell J, Judge A, Cooper C, Prieto-Alhambra D, Arden NK et al (2015) Future projections of total hip and knee arthroplasty in the UK: results from the UK Clinical Practice Research Datalink. Osteoarthr Cartil 23:594-600

13. Dawson J, Fitzpatrick R, Murray D, Carr A (1998) Questionnaire on the perceptions of patients about total knee replacement. J Bone Jt Surg Br 80-B:63-69

14. Dixon S, Blom AW, Whitehouse MR, Wylde V (2014) Comparison of patient reported outcomes after Triathlon $((\mathrm{R}))$ and Kinemax Plus prostheses. Ann R Coll Surg Engl 96:61-66

15. Dunbar MJ, Robertsson O, Ryd L, Lidgren L (2001) Appropriate questionnaires for knee arthroplasty. Results of a survey of 3600 patients from the Swedish Knee Arthroplasty Register. J Bone Joint Surg Br 83:339-344

16. Eckhoff DG, Bach JM, Spitzer VM, Reinig KD, Bagur MM, Baldini TH et al (2003) Three-dimensional morphology and kinematics of the distal part of the femur viewed in virtual reality. Part II. J Bone Jt Surg Am 85-A(Suppl 4):97-104
17. Ewald FC (1989) The Knee Society total arthroplasty roentgenographic evaluation and scoring system. Clin Orthop Relat Res 248:9-12

18. Frankel VH, Burstein AH, Brooks DB (1971) Biomechanics of internal derangement of the knee. pathomechanics as determined by analysis of the instant centers of motion. J Bone Jt Surg Am 53:945-962

19. Grieco TF, Sharma A, Komistek RD, Cates HE (2016) Single versus multiple-radii cruciate-retaining total knee arthroplasty: an in vivo mobile fluoroscopy study. J Arthroplast 31:694-701

20. Hamilton DF, Burnett R, Patton JT, Howie CR, Moran M, Simpson $\mathrm{AH}$ et al (2015) Implant design influences patient outcome after total knee arthroplasty: a prospective double-blind randomised controlled trial. Bone Jt J 97-B:64-70

21. Hamilton DF, Loth FL, Giesinger JM, Giesinger K, MacDonald DJ, Patton JT et al (2017) Validation of the English language Forgotten Joint Score-12 as an outcome measure for total hip and knee arthroplasty in a British population. Bone Jt J 99-B:218-224

22. Hamilton DF, Simpson AHRW, Burnett R, Patton JT, Moran M, Clement ND et al (2013) Lengthening the moment arm of the patella confers enhanced extensor mechanism power following total knee arthroplasty. J Orthop Res 31:1201-1207

23. Harwin SF, Greene KA, Hitt K (2008) Triathlon total knee arthroplasty: 4 year outcomes with a high performance implant. J Knee Surg 21:320-326

24. Hinarejos P, Puig-Verdie L, Leal J, Pelfort X, Torres-Claramunt R, Sanchez-Soler J et al (2016) No differences in functional results and quality of life after single-radius or multiradius TKA. Knee Surg Sports Traumatol Arthrosc 24:2634-2640

25. Hollister AM, Jatana S, Singh AK, Sullivan WW, Lupichuk AG (1993) The axes of rotation of the knee. Clin Orthop Relat Res 259-268

26. Howell SM, Howell SJ, Hull ML (2010) Assessment of the radii of the medial and lateral femoral condyles in varus and valgus knees with osteoarthritis. J Bone Jt Surg Am 92:98-104

27. Inacio MCS, Graves SE, Pratt NL, Roughead EE, Nemes S (2017) Increase in total joint arthroplasty projected from 2014 to 2046 in Australia: a conservative local model with international implications. Clin Orthop Relat Res 475:2130-2137

28. Insall J, Tria AJ, Scott WN (1979) The total condylar knee prosthesis: the first 5 years. Clin Orthop Relat Res 145:68-77

29. Jenkins PJ, Clement ND, Hamilton DF, Gaston P, Patton JT, Howie CR (2013) Predicting the cost-effectiveness of total hip and knee replacement: a health economic analysis. Bone Jt J 95-B:115-121

30. Kim J, Nelson CL, Lotke PA (2004) Stiffness after total knee arthroplasty. Prevalence of the complication and outcomes of revision. J Bone Jt Surg Am 86-A:1479-1484

31. Kurtz SM, Lau E, Ong K, Zhao K, Kelly M, Bozic KJ (2009) Future young patient demand for primary and revision joint replacement: national projections from 2010 to 2030. Clin Orthop Relat Res 467:2606-2612

32. Nilsdotter AK, Toksvig-Larsen S, Roos EM (2009) Knee arthroplasty: are patients' expectations fulfilled? A prospective study of pain and function in 102 patients with 5-year follow-up. Acta Orthop 80:55-61

33. Palmer J, Sloan K, Clark G (2014) Functional outcomes comparing Triathlon versus Duracon total knee arthroplasty: does the Triathlon outperform its predecessor? Int Orthop 38:1375-1378

34. Scott CE, Clement ND, Macdonald DJ, Hamilton DF, Gaston P, Howie CR et al (2015) Five-year survivorship and patientreported outcome of the Triathlon single-radius total knee arthroplasty. Knee Surg Sports Traumatol Arthrosc 23:1676-1683

35. Scott CE, Oliver WM, MacDonald D, Wade FA, Moran M, Breusch SJ (2016) Predicting dissatisfaction following total knee arthroplasty in patients under 55 years of age. Bone Jt J 98-B:1625-1634 
36. Scott CEH, Howie CR, MacDonald D, Biant LC (2010) Predicting dissatisfaction following total knee replacement: a prospective study of 1217 patients. J Bone Jt Surg Br 92-B:1253-1258

37. Stambough JB, Clohisy JC, Barrack RL, Nunley RM, Keeney JA (2014) Increased risk of failure following revision total knee replacement in patients aged 55 years and younger. Bone Jt J 96-B:1657-1662
38. van Jonbergen HP, Reuver JM, Mutsaerts EL, Poolman RW (2014) Determinants of anterior knee pain following total knee replacement: a systematic review. Knee Surg Sports Traumatol Arthrosc 22:478-499

39. Williams DP, Blakey CM, Hadfield SG, Murray DW, Price AJ, Field RE (2013) Long-term trends in the Oxford knee score following total knee replacement. Bone Joint J 95-B:45-51 